\title{
ViVa: The Virtual Vascular Project
}

\author{
Gassan Abdoulaev $^{\dagger}$, Sandro Cadeddu ${ }^{\dagger}$, Giovanni Delussu ${ }^{\dagger}$,

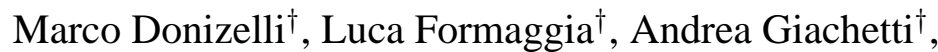 \\ Enrico Gobbetti ${ }^{\dagger}$, Andrea Leone ${ }^{\dagger}$, Cristina Manzi ${ }^{\dagger}$, \\ Piero Pili ${ }^{\dagger}$, Alan Scheinine ${ }^{\dagger}$, Massimiliano Tuveri ${ }^{\dagger, \ddagger}$, \\ Alberto Varone ${ }^{\dagger}$, Alessandro Veneziani ${ }^{\star}$, \\ Gianluigi Zanetti ${ }^{\dagger}$, and Antonio Zorcolo ${ }^{\dagger}$ \\ $\dagger$ CRS4, Via Sauro 10, 09123 Cagliari, Italy \\ $\ddagger$ Clinica Sant’Elena, Cagliari, Italy \\ * Facoltà di SS. MM. FF. NN., Università di Verona, Verona, Italy \\ E-mail: zag@crs4.it
}

To appear in: IEEE Transactions on Information Technology in Biomedicine, Vol 22, No. 4, Dec. 1998.

\begin{abstract}
The aim of the ViVa project is to develop tools for the modern hemodynamicist and cardiovascular surgeon to study and interpret the constantly increasing amount of information being produced by non-invasive imaging equipment. In particular, we are developing a system able to process and visualize $3 D$ medical data, to reconstruct the geometry of arteries of specific patients and to simulate blood flow in them. The initial applications of the system will be for clinical research and training purposes. In a later stage we will explore the application of the system to surgical planning. ViVa is based on an integrated set of tools, each dedicated to a specific aspect of the data processing and simulation pipeline: image processing and segmentation; real-time $3 D$ volume visualization; $3 D$ geometry reconstruction; $3 D$ mesh generation; blood flow simulation and visualization.
\end{abstract}

\section{Goals and Motivation}

Recent advances in non-invasive data acquisition technology, e.g., Spiral Computerized Tomography and fast Magnetic Resonance Imaging, are now providing physicians with large amounts of quantitative information on a patient's body internal structures. The availability of fast computers will make it possible to harness this information to provide surgeons with accurate $3 \mathrm{D}$ reconstructions and with the ability of using simulation for training purposes and to foresee the effects of surgical intervention.

In the cardiovascular system, morphology and functionality are closely related. Altered flow conditions, such as separation, recirculating zones and oscillatory shear stress, play an important role in the development of arterial diseases. In turn all these flow conditions are modified by arterial wall changes such as intimal thickening or atherosclerotic plaques. A detailed understanding of the influence of wall modifications on flow patterns can have useful surgical applications, especially towards reconstruction and revascularization problems. Furthermore, the ability of simulating vascular operations on an 
accurate virtual model of the vessel tree will allow surgeons to anticipate the effects on blood flow of modifications in the vessel geometry due, for instance, to the insertion of prosthesis, such as a stent, or to the addition of new vessel junctions, e.g. a bypass (see e.g. [1]).

The aim of the Virtual Vascular project (ViVa) is to develop tools for the modern hemodynamicist and cardiovascular surgeon to study and interpret the constantly increasing amount of information being produced by noninvasive imaging equipment. In particular, we are developing a system that will be able to process and visualize 3D medical data, reconstruct the geometry of arteries of specific patients and to simulate blood flow in them. A similar project, the "Computational Vascular Laboratory" (CVL), is being actively pursued at Stanford. The initial applications of ViVa will be for clinical research and training purposes, while CVL has a stronger emphasis on surgical planning issues[2]. In a later stage we will also explore the application of the system to surgical planning as well as the integration with global, but less detailed, cardiovascular models such as the one developed within SIMA, a project whose goal is to develop a fully fledged anesthesiology simulator based on explicit mathematical modeling[3].

$\mathrm{ViVa}$ is a multi-disciplinary project that involves expertise from many research areas, ranging from physiology to numerical analysis. ViVa and SIMA are partially funded by the EUREKA project EU 1063 (HPPC-SEA). CRS4 is the HPPC-SEA partner responsible for ViVa, while MathTech Asp. (Copenhagen, Denmark) is responsible for SIMA. This short paper provides an overview of ViVa current research activities.

\section{Research Overview}

ViVa is based on an integrated set of tools, each dedicated to a specific aspect of the data processing and simulation pipeline: image processing and segmentation; realtime 3D volume visualization; 3D geometry reconstruction; 3D mesh generation; blood flow simulation and visualization.

ViVa focuses on providing a detailed 3D description of local aspects of vascular geometries and blood flow, for instance in the vicinity of a pathological region such as a stenosis. Figure 1 gives a graphical representation of the project; the following sections summarize our activities and results in the different areas.

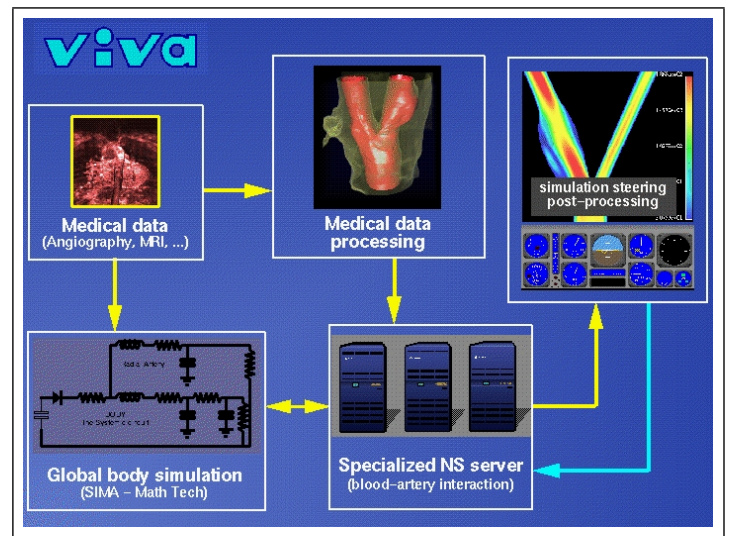

Figure 1: ViVa Overview: all the research areas are shown

\subsection{Data acquisition}

In recent years there has been an increasing interest in the use of computational fluid dynamics simulation for the qualitative study of cardiovascular problems. The majority of current simulations are based on simplified geometrical models that represent "typical" descriptions of the anatomy of the region under examination. Even though these simulations capture many interesting features of the flow structure, it is difficult to apply these results to actual clinical problems, since flow behavior depends strongly on details of the vessel geometry and there is a large variability of the latter in the population[4]. Recent advances in medical imaging techniques $[5,6]$ promise to dramatically improve the situation, since it is becoming feasible to acquire in a non-invasive manner not only the vessel geometry but also gross informations on the blood flow itself.

Thus, one of the crucial aspects of ViVa is the ability of directly interfacing with data-acquisition modalities such as CT and MR scanners. The medical data we use is stored on our CTN image server[7] and it is provided by research collaborations with clinical research institutions. Data transmission between the hospitals and our PACS is usually performed using DICOM. In this context, we have experimented with the Italian Research ATM backbone 
(Syrius) for data transfer from/to the Radiology Dept. of the University of Pisa [8] and we are using a dedicated 2 Mbit/s line between our laboratory and the San Michele Hospital.

The dataset used thus far include patients and volunteers data, "in-vitro" MR velocity measurements in reference geometries[9], as well as high-resolution CT scans of autoptic arterial specimens.

\subsection{Image processing and segmentation}

The purpose of the image processing and segmentation subsystems is to extract geometrical information from CT and MR scans using several segmentation algorithms, controlled by a specifically designed user interface. Our segmentation system uses a 2D edge follower, snakes[10] and balloon[11] models (see figure 2). The 2D contours obtained from the segmentation step are then connected to reconstruct a 3D model of the arteries (see section on geometric modeling). For a similar approach see [12].

The system is is based on a distributed implementation with user interfaces clients and dedicated server processes for DICOM image retrieval and processing. It is characterized by an object-oriented client-server architecture that provides a complete framework for the interactive segmentation of CT or MR scans of carotid artery sections using the Generalized Active Contour Model (GACM)[13]. The client side of the system is implemented with a graphical user interface (GUI) written in Java, while a GACM-based algorithm written in $\mathrm{C}++$ is included in the server side. The client and the server are connected through a CORBA communication infrastructure, i.e., through a distributed object computing middleware [14].

\subsection{D volume visualization}

In order to analyze acquired volumetric data, we are currently developing a system that merges the concepts of interactive direct volume rendering and virtual endoscopy in a tool for non-invasive analysis of diseased vessels. Our interactive virtual angioscopy system takes as input data acquired with standard medical imaging modalities and regards it as a virtual environment to be interactively inspected. The system supports real-time navigation with

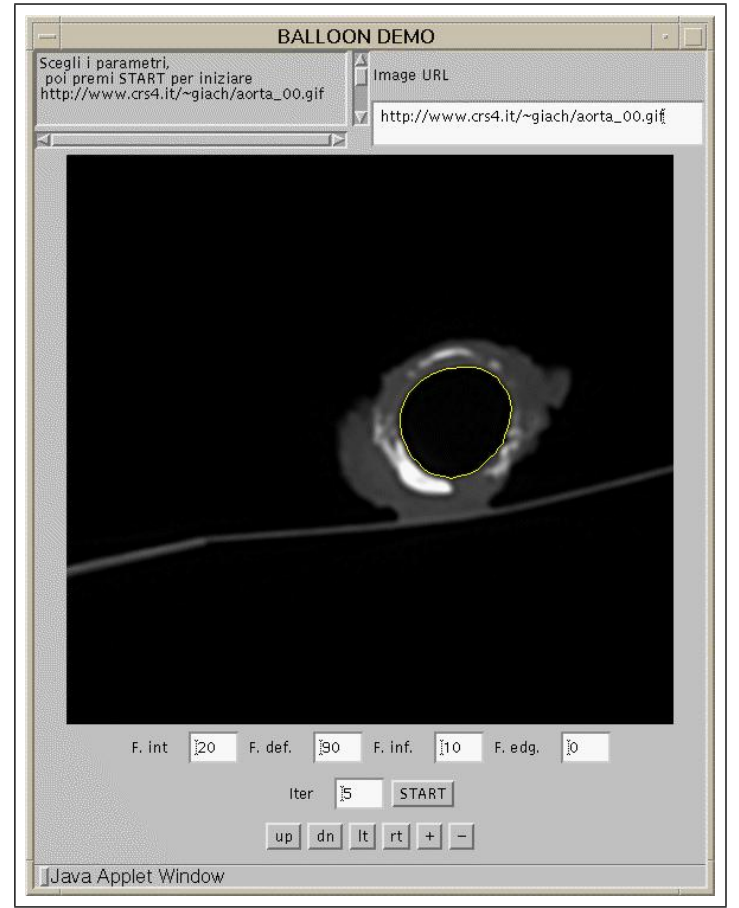

Figure 2: User interface of the image processing and segmentation subsystem

stereoscopic direct volume rendering and dynamic endoscopic camera control, interactive tissue classification, and interactive point picking for morphological feature measurement $[15,16]$.

When the dataset is loaded on the graphical workstation, the system presents a perspective view of the dataset and users may interactively take morphological measures, move the viewpoint, and modify the classification parameters to obtain the best visual representation of the particular anatomy under examination.

In our application, the user interacts with the rendered volume using both hands simultaneously: the left hand, controlling a Spaceball, is used for 3D navigation with six-degree-of-freedom control, while the right hand, holding a mouse, is used to operate two-dimensional widgets and to measure morphological features by picking points on the volume (Figure 3). A simple 2D user interface in a separate panel is used for specifying the transfer function mapping data values to opacity/color values [17]. This 


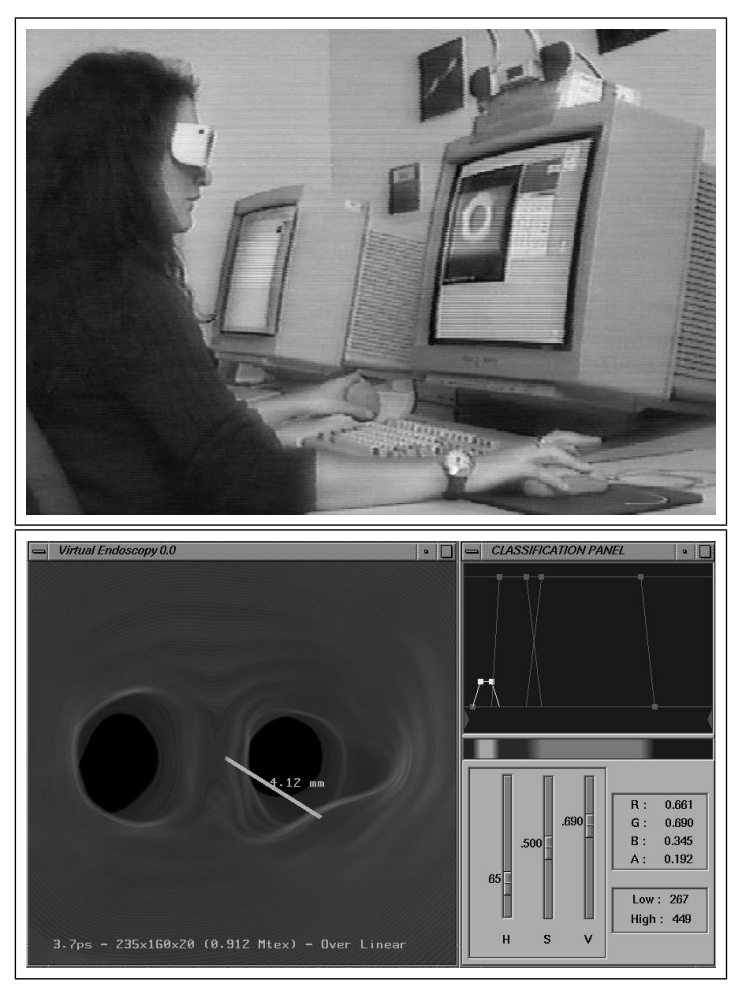

Figure 3: Top: Interactive session with the virtual angioscopy system, Bottom: Graphical user interface of the virtual angioscopy system

way it is possible to interactively extract from the original data the required morphological features.

The system aims at supporting vascular surgeons in the understanding of the precise spatial location and shape of the vessel lesions, which is of primary importance for vascular disease treatment. The tests made in collaboration with vascular surgeons have given promising results. Diseased areas have been easily identified and important morphological features have been measured (figure 4) $[15,16]$. For validation purposes, we are currently in the process of comparing the measures taken with our virtual angioscopy prototype with what can be obtained by actually measuring morphometric data of explanted vessels after projecting them onto a digitizing plate and then storing in a computer for the comparison.

Virtual angioscopy promises to be a non-invasive medical procedure that can improve the diagnostic sensitivity

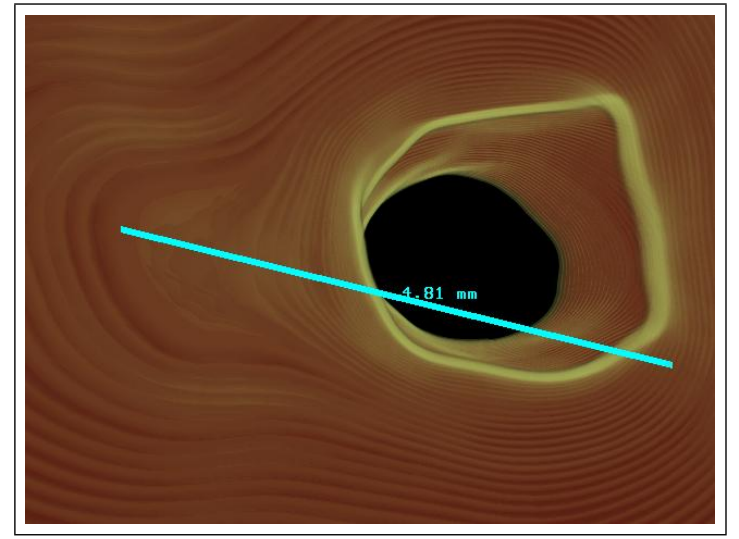

Figure 4: Endoscopic view of a stenosis area inside a human right carotid artery (autoptic specimen, 256x256x32 CT dataset, 12 bit resolution, depth shading, Drebin classification). The surgeon has identified the stenosis area and interactively measures the variation in lumen of the vessel. The ruler is interactively dragged during examination, and the endpoints automatically follow the interior surface of the vessel. Here, the ruler is placed before the stenosis, measuring the lumen of the vessel.

in the vascular domain. Currently, the system requires a high-end graphics workstation to provide enough performance for being used for real clinical studies. Expansion of the PC and video game markets means that good texture mapping hardware accelerators may soon make these methods affordable in real medical settings.

\subsection{D geometry reconstruction}

A good description of arterial geometries is one of the essential ingredients of the ViVa project. Vessel geometries are the objects that doctors will manipulate during surgical planning and they are the natural starting points for vascular fluid dynamics simulations. The description of vessel geometries is handled by the Geometric Modeling Engine module (GME).

The basic idea of GME is to guarantee a sufficient level of abstraction between vessel geometrical entities and the rest of the system. Thus, it is GME responsibility to present to other ViVa modules a description of the arterial tree suitable for the interactive re-connection of arteries 
in a simulated by-pass operation, and the computation of the local curvature tensor of the vessel internal surfaces for automatic computational mesh spacing determination. Currently, we are using the GME module mainly for the $3 \mathrm{D}$ reconstruction of vessel trees. We are extending our geometrical modeling tool to support interactive manipulation of vessel geometries. This kind of extension is critical since our experience indicates that the time involved in actual computation, on modern workstations and highend PCs, is negligible with respect to the time required by human interaction.

The core of our GME is based on Shapes, a geometric computing kernel library developed by Xox Corp[18]. Its geometrical and topological framework is a kind of Boundary Representation (BRep) that has been proven very powerful in this context, see also [2].

Figure 5 shows the geometric reconstruction of a human carotid.

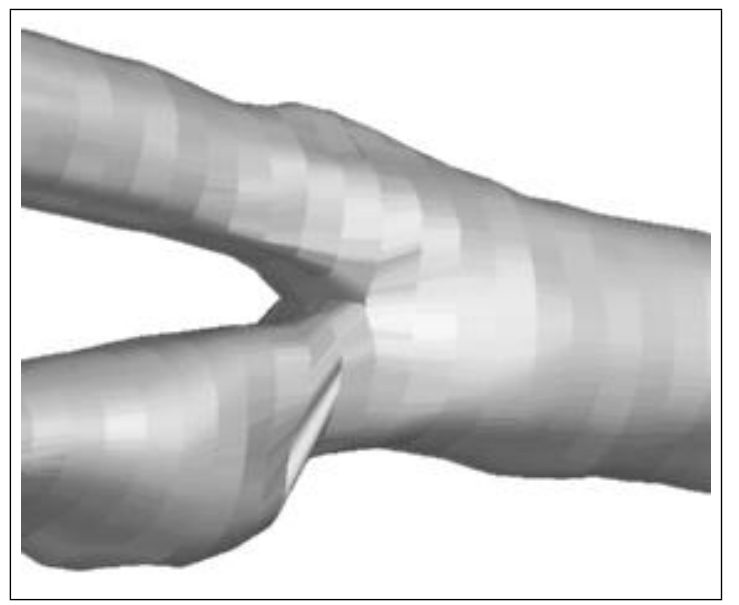

Figure 5: 3D geometric model reconstructed from contours obtained by segmenting using GACM the CT images of an autoptic specimen of a carotid bifurcation segmentation

\subsection{D mesh generation}

The purpose of MGS, the 3D mesh generation subsystem, is to provide ViVa with flexible tools for the generation of 3D unstructured and hybrid meshes on complex vessel geometries, in particular in the bifurcations regions. Accu- rate numerical simulations of a physical phenomenon rely on the quality of the computational domain discretization. Thus, a good mesher allows the simulator to save time and resources or simply to obtain better results.

Even if commercial mesh generation codes are available nowadays, they often do not offer the flexibility of an especially designed program, and their integration within multidisciplinary applications is generally complex. Moreover, our grid generation system is designed to be well integrated with the geometrical modeling engine described in the previous section and with the special purpose multi-domain Navier Stokes solver being developed within ViVa, see next section.

MGS supports a variety of geometrical representation, limited, however, to compact, orientable, two-manifold surfaces and to orientable curves. GSM interfaces to the topological and geometrical description via an abstract interface either to GME, or to an alternative simplified geometric modeler. In the B-Rep model used internally by MGS the various objects are represented by means of their boundaries. In particular, 3D solids are represented by means of their enclosing surfaces. The model is hierarchical in the sense that the surfaces are in turn represented by means of their enclosing curves (loops) and so on. The geometry, that is the actual description of the domain shape, is represented by a collection of points, curves and surfaces, while the topology, that is the description of how the geometrical elements are joined to form the global model, is constituted by vertices, edges, loops, faces, shells, blocks.

The actual mesh generator module of MGS is a collection of routines which provide different methodologies for generating meshes of every dimensionality i.e. on edges, faces and blocks [19]. Presently, we have integrated in the library generation procedures based on the front advancing method. We expect to implement other routines which make use of the constrained Delaunay method. The postprocessing module will implement mesh adaption routines with the goal of increasing or decreasing the mesh density according to user prescribed criteria and local geometrical features such as surface curvature. The Visualization module will provide graphical support and allow the real-time display of mesh generation and geometry creation. The final code will support non/conforming multilevel grids, hybrid meshes as well as contain tools for multilevel grid generation. 


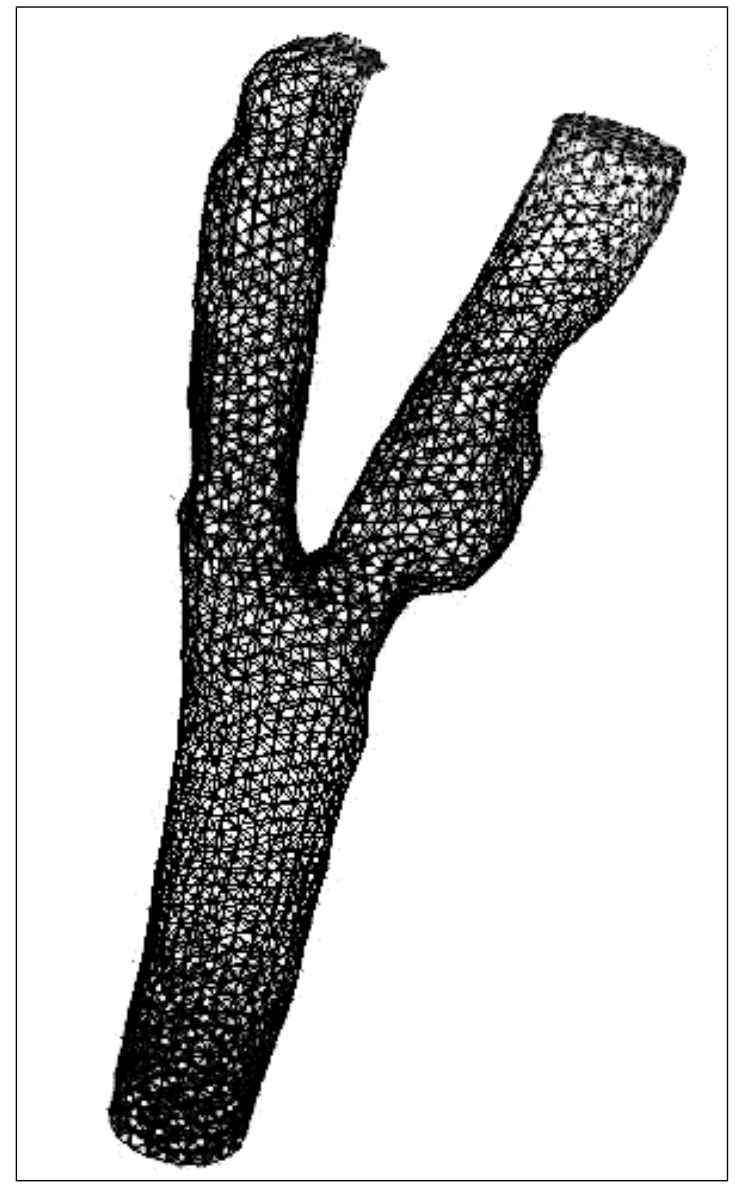

Figure 6: A 3D computational mesh generated from the geometry shown in figure 5

\subsection{Blood flow simulation}

The purpose of this subsystem is mainly to support vascular flow simulation models able to complement, by extrapolation and/or interpolation, experimental information acquired by various medical apparata. As an example of extrapolation, we can mention the use of a simulation of flow in a vessel to extrapolate experimentally unaccessible quantities, such as the shear stress at the vessel wall, from measured quantities, such as blood flow velocity profiles obtained by fast RM velocimetry [6] (see also [20, 21]). Interpolation is instead mostly related to parametric studies, e.g., estimating the effects of variation in the angle of a new vessel junction geometry on blood flow.

To be clinically relevant, the system should be able to deal with complex vessels geometries and to provide the results of simulations "over-night" on high-end workstations. The first requirement comes from the necessity of working with vessel models derived from imaging data relative to the actual patient under study, while the latter from typical clinical work-flow constraints.

The subsystem includes a flow solver, specialized grid generation and flow visualization modules. The blood flow simulation activity within $\mathrm{ViVa}$ is based on using a succession of simulation platforms. The present platform uses for the blood flow solver commercial packages such as Simulog N3S and Centrics Spectrum. However, in order to meet efficiency constraints we are developing a dedicated blood flow solver. We have chosen a domain decomposition strategy, based on the mortar element method [22], which allows the simulator to utilize non matching meshes in different sub-domains. As a result, we are able to separate areas with complex geometry and flow patterns, and to use there meshes fine enough to approximate with a good accuracy flow fields and pressure distributions, without affecting areas where the solution is smooth and coarser meshes can be used. Moreover, simpler sub-domain shapes allow the utilization of fast algebraic multilevel method with resulting gains in computational speed. The time-dependent Navier-Stokes solver takes advantage of the fractional step projection method coupled with the Lagrangian-Galerkin procedure for the convective term. The combination of the numerical techniques mentioned above has been proved to be very efficient, particularly for multiprocessor computers, for a detailed description see [23].

The current blood flow simulation activity is concentrated on investigating the flow solution accuracy and its dependence on geometrical reconstruction errors. For instance, we are comparing simulation data with data coming from an experimental set-up based on in-vitro measurement of flow in anastomosis glass replicas imaged using a 7T MRI equipment of the MR Research Centre, Skejby Sygehus, Aarhus University Hospital, Denmark [9]. The acquired data are 3D morphological images giving the complete geometry of the replicas and 3D velocity field information on selected planes. From the morphological data we reconstruct a computer model of the vessel 
internal geometry. Then, starting from the latter, we build a computational grid, and, finally, run fluid flow simulation. The computed flow in the reconstructed geometry is in good qualitative agreement with the measured data and the preliminary results obtained on these anastomosis geometries show that simulations can be used to extrapolate more information from noisy measures and to analyze flow patterns [9]. Fig.7 gives an example of a simulation result.

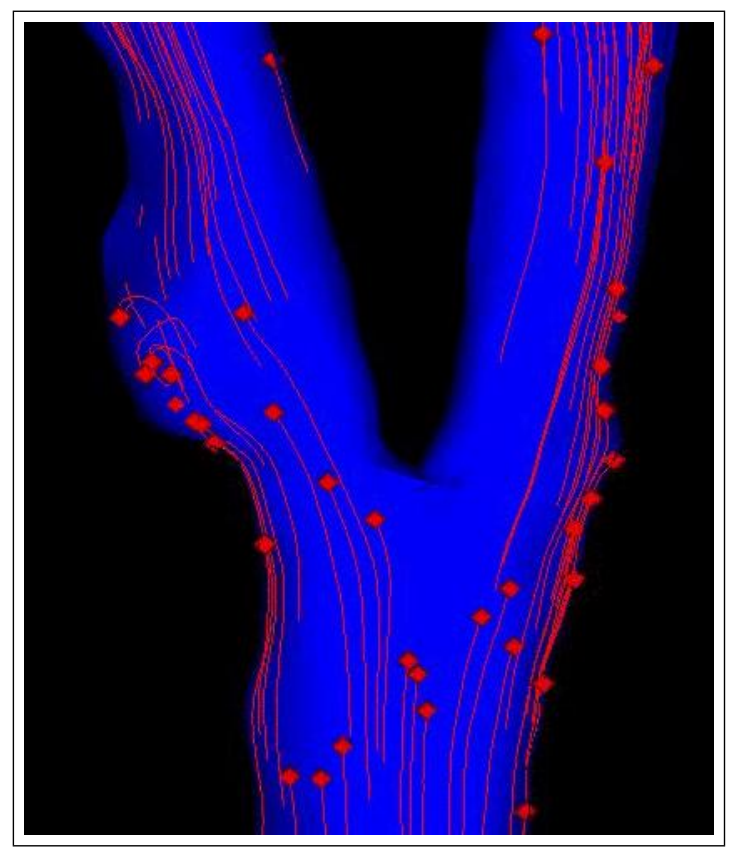

Figure 7: Simulated blood flow patterns in the arterial model shown in figure 5. Note the complex 3D flow structure in the carotid sinus region (on the left)

\section{Conclusions}

We have presented our research efforts aimed at developing tools for the modern hemodynamicist and cardiovascular surgeon to study and interpret the constantly increasing amount of information being produced by noninvasive imaging equipment. In particular, we are developing a system able to process and visualize 3D medical data, reconstruct the geometry of arteries of specific pa- tients and simulate blood flow in them. Prototypes have already been developed for each of the specific aspects of the data processing and simulation pipeline: image processing and segmentation; real-time 3D volume visualization; 3D geometry reconstruction; 3D mesh generation; blood flow simulation and visualization.

Understanding the local hemodynamic environment in a region of the vascular system is an important field of research. There is, in fact, a strong positive correlation between arterial wall modifications and the hemodynamic environment. Some of these alterations are not well understood, providing enormous difficulties to foresee shortand long-term evolution of the atherosclerotic disease and thus planning the most correct approach. This system offers the possibility to study the arterial alterations, to plan surgical procedures on the basis of the morphological and functional findings, and, hopefully, to timely correct wrong surgical results, if any.

Besides their interest for clinical research and surgical planning, "virtual" vessel models can also be used as the basis for sophisticated training systems. As an example, we can consider the simulation of angioplastic procedures, one of the foreseen applications of this research project. Angioplasty is a set of surgical procedures whose goal is to cure vascular pathologies, such as stenosis, from within the vessel lumen. The procedures are based on the insertion of a catheter in the patient artery, the positioning of the catheter by navigating the arterial tree, and the final inflation of a balloon in the stenotic region to enlarge the vessel lumen. It is our opinion that many aspects of angioplasty procedures can be effectively reproduced with a dedicated simulation system based on detailed three-dimensional virtual models of the vascular tree augmented by a physically based computational model of the interaction between the catheter, the blood and the vessel walls. A virtual, patient-specific environment offers different options to the operator (resident or student, vascular surgeon or radiologist) approaching this complex medical field: first, the understanding of the intimate correlation between any modification of vessel morphology and the associated alteration of flow patterns, with its repercussions on long-term results; second, the basis for a sophisticated training, before performing procedures on actual patients; last, the possibility to alter vascular geometries in order to study or foresee end-results of procedures. 


\section{Acknowledgments}

The medical data sets used within ViVa are provided by research collaborations with: the Radiology Dept. of the San Michele Hospital, Cagliari, Italy; Radiology Dept., University of Pisa, Pisa, Italy; Vascular and Thoracic Surgery, Skejby Sygehus, Aarhus University Hospital, Denmark.

The results presented here were obtained within the VIrtual VAscular project (ViVa), a large multidisciplinary project at CRS4, whose main objective is to develop simulators for vascular applications. ViVa is partially funded by the EUREKA project EU 1063. We also acknowledge the contribution of Sardinian regional authorities.

\section{References}

[1] F. Loth, S.A. Jones, D.P. Giddens, H.S. Bassiouny, S. Glagov, and C.K. Zarins, "Measurements of velocity and wall shear stress inside a ptfe vascular graft model under steady flow conditions", $J$ Biomech Eng, vol. 119, pp. 187-194, 1997.

[2] C. A. Taylor, T. J. R. Hughes, and C. K.Zarins, "Computational investigations in vascular disease", Computers in Physics, vol. 10, pp. 224-232, 1997.

[3] J. Larsen and S. A. Pedersen, "The mathematical models behind advanced models in medicine", in J.T. Ottosen and M. Danielsen, editors, Mathematical Modelling in Medicine. Roskilde University Press, (In press), 1998.

[4] C.G. Caro, D.J. Doorly, M. Tarnawski, K.T. Scott, Q. Long, and C.L. Dumoulin, "Non-planar curvature and branching of arteries and non-planar-type flow", Proc. R. Soc. London, vol. 452, pp. 185-197, 1996.

[5] W.Y. Kim, P.G. Walker, E.M. Pedersen, J.K. Poulsen, S. Oyre, K. Houlind, and A.P. Yogonathan, "Left ventricular blood flow patterns in normal subjects: A quantitative analysis by three dimensional magnetic resonance velocity mapping", Journal of American Coll. Card., vol. 26, pp. 224-238, 1995.
[6] D.N. Ku, C. L. Biancheri, R. I. Pettigrew, J. W. Peifer, C. P. Markou, and H. Engels, "Evaluation of magnetic resonance velocimetry for steady flow", J. Biomech. Eng., vol. 112, pp. 464, 1990.

[7] Washington University, MIR CTN documentation Version 2.8.3.

[8] A. Giachetti and C. Petri, "Medical image transmission on heterogeneous network", in C. Bortolazzi and D. Caramella, editors, Proceedings Of The 15th Europacs Annual Meeting, pp. 117-120, September 1997.

[9] A. Giachetti, G. Zanetti, S. Cadeddu, M. Tuveri, NH. Staalsen, and S. Ringgaard, "Comparison between numerical flow simulations and $\mathrm{mr}$ measures on glass anastomosis models", in VIII Mediterranean Conference on Medical and Biological Engineering and Computing (Medicon98), 1998.

[10] M. Kass, A. Witkin, and D. Terzopoulos, "Snakes: Active contour models", Int. Journal of Computer Vision, vol. 1, pp. 321-331, 1987.

[11] L. Cohen, "On active contour models and balloons", CVGIP: Image Understanding, vol. 53, pp. 211218, Mar. 1991.

[12] Q. Long, X. Y. Xu, M. W. Collins, M. Bourne, and T. M. Griffith, "Generation of cfd velocity boundary condition from cine $\mathrm{mr}$ phase-contrast image", in Proceedings of Medical Image Understanding and Analysis '97, pp. 117-120, University of Oxford, 1997.

[13] K. F. Lai and R. T. Chin, "Deformable contours: Modelling and extraction", IEEE Transactions on Pattern Analysis and Machine Intelligence, vol. 17, pp. 1084-1090, 1995.

[14] A. Scheinine, M. Donizelli, and M. Pescosolido, "An object-oriented client-server system for interactive segmentation of medical images using the method of active contours", in Image Processing for the Medical Sciences, Held in Aachen, Germany, March 26-27, 1998. 
[15] Piero Pili, Antonio Zorcolo, Enrico Gobbetti, and Massimiliano Tuveri, "Interactive 3D visualization of carotid arteries", International Angiology, vol. 16, pp. 153, Sep. 1997.

[16] Enrico Gobbetti, Piero Pili, Antonio Zorcolo, and Massimiliano Tuveri, "Interactive virtual angioscopy", in Proceedings IEEE Visualization, Conference held in Research Triangle Park, NC, USA, Oct. 1998 (In press).

[17] Robert A. Drebin, Loren Carpenter, and Pat Hanrahan, "Volume rendering", Computer Graphics, vol. 22, pp. 65-74, Aug. 1988.

[18] Xox Corp., Shapes Kernel, A system For Computing with Geometrics Objects, Reference Manual, release 2.1.5 edition.

[19] K.J. Weiler, "Edge-based data structures for solid modelling in curved surface environments", Computer Graphics and Applications, vol. 5, pp. 25-37, Jan. 1985.

[20] K. Perktold and G. Rappitsch, "Computer simulation of local blood flow and vessel mechanics in a compliant carotid artery model", J. Biomechanics, vol. 28, pp. P845-856, 1995.

[21] Q. Long, XY Xu, and MW Collins, "Generation of structure of the aortic bifurcation from magnetic resonance angiograms", in M. Cerrolaza, D. Jugo, and CA Brebbia, editors, Proc. of The first Inter. conference on simulation modelling in bioengineering, BIOSIM 96, pp. 217-226, 1996.

[22] C.Bernardi, Y.Maday, and A.Patera, "A new nonconforming approach to domain decomposition: the mortar element method", in H.Brezis and J.L.Lions, editors, Nonlinear Partial Differential Equations and Their Applications. Pitman, 1993.

[23] G.S.Abdoulaev, Y.Achdou, J.-C.Hontand, Yu.A.Kuznetsov, O.Pironneau, and C.Prudhomme, "Nonmatching grids for fluids", in Proc. of the 10th Intern. Conf. on Domain Decomposition Methods, Held in Boulder, August 10-14, 1997. 